\title{
Community Detection in Quantum Complex Networks
}

\author{
Mauro Faccin, ${ }^{1, *}$ Piotr Migdał, ${ }^{2,1}$ Tomi H. Johnson, ${ }^{3,4,5,1}$ Ville Bergholm, ${ }^{1}$ and Jacob D. Biamonte ${ }^{1}$ \\ ${ }^{1}$ ISI Foundation, Via Alassio 11/c, 10126 Torino, Italy \\ ${ }^{2}$ ICFO-Institut de Ciències Fotòniques, 08860 Castelldefels (Barcelona), Spain \\ ${ }^{3}$ Centre for Quantum Technologies, National University of Singapore, \\ 3 Science Drive 2, 117543, Singapore \\ ${ }^{4}$ Clarendon Laboratory, University of Oxford, Parks Road, Oxford OX1 3PU, United Kingdom \\ ${ }^{5}$ Keble College, University of Oxford, Parks Road, Oxford OX1 3PG, United Kingdom
}

(Received 6 May 2014; revised manuscript received 11 July 2014; published 21 October 2014)

\begin{abstract}
Determining community structure is a central topic in the study of complex networks, be it technological, social, biological or chemical, static or in interacting systems. In this paper, we extend the concept of community detection from classical to quantum systems - a crucial missing component of a theory of complex networks based on quantum mechanics. We demonstrate that certain quantum mechanical effects cannot be captured using current classical complex network tools and provide new methods that overcome these problems. Our approaches are based on defining closeness measures between nodes, and then maximizing modularity with hierarchical clustering. Our closeness functions are based on quantum transport probability and state fidelity, two important quantities in quantum information theory. To illustrate the effectiveness of our approach in detecting community structure in quantum systems, we provide several examples, including a naturally occurring light-harvesting complex, LHCII. The prediction of our simplest algorithm, semiclassical in nature, mostly agrees with a proposed partitioning for the LHCII found in quantum chemistry literature, whereas our fully quantum treatment of the problem uncovers a new, consistent, and appropriately quantum community structure.
\end{abstract}

DOI: 10.1103/PhysRevX.4.041012

\section{INTRODUCTION}

The identification of the community structure within a network addresses the problem of characterizing the mesoscopic boundary between the microscopic scale of basic network components (herein called nodes) and the macroscopic scale of the whole network [1-3]. In nonquantum networks, the detection of community structures dates back to Rice [4]. Such analysis has revealed countless important hierarchies of community groupings within realworld complex networks. Salient examples can be found in social networks such as human [5] or animal relationships [6], biological [7-10], biochemical [11], and technological $[12,13]$ networks, as well as numerous others (see Ref. [1]). In quantum networks, as researchers explore networks of an increasingly nontrivial geometry and large size [14-17], quantum networks analysis and understanding will involve identifying nontrivial community structures. In this article, we devise methods to perform this task, providing an important missing component in the recent drive to unite quantum physics and complex network science $[18,19]$.

\footnotetext{
*mauro.faccin@isi.it
}

Published by the American Physical Society under the terms of the Creative Commons Attribution 3.0 License. Further distribution of this work must maintain attribution to the author(s) and the published article's title, journal citation, and DOI.
Subject Areas: Complex Systems, Quantum Physics

For quantum systems, beyond being merely a tool for analysis following simulations, community partitioning is closely related to performing the simulations themselves. Simulation is generally a difficult task [20], e.g., simulating exciton transport in dissipative quantum biological networks [21-26]. The amount of resources required to exactly simulate such processes scales exponentially with the number of nodes. To overcome this, one must in general seek to describe only limited correlations between certain parts of the network [27]. Mean-field [28-31] and tensornetwork methods [32,33] assume correlations between bipartitions of the system along some node structure to be zero or limited by an area law. Hartree-Fock methods assume limited correlations between particles [34,35]. Thus, planning a simulation involves identifying a partitioning of a system for which it is appropriate to limit intercommunity correlations; i.e., it is a type of community detection.

We apply our detection methods to artificial networks and the real-world light-harvesting complex II (LHCII) network. In past works, researchers have divided the LHCII by hand in order to gain more insight into the system dynamics [36-38]. Meanwhile, our methods optimize the task of identifying communities within a quantum network $a b$ initio and the resulting communities consistently point towards a structure that is different from those previously identified for the LHCII. For larger networks, as with our 
artificial examples, automatic methods appear to be the only feasible option.

Our specific approach is to generate a hierarchical community structure [39] by defining both internode and intercommunity closeness. The optimum level in the hierarchy is determined by a modularity-based measure, which quantifies how good a choice of communities is for the quantum network on average relative to an appropriately randomized version of the network. Although modularity-based methods are known to struggle with large sparse networks [40,41], this work focuses on quantum systems whose size remains much smaller than the usual targets of classical community detection algorithms.

While the backbone of our quantum community method is shared with classical methods, the physical properties used to characterize a good community in a quantum must necessarily be very different from the properties used for a classical system. Here, we show how two quantum properties are used to obtain closeness and modularity functions: the first is the coherent transport between communities and the second is the change in the states of individual communities during a coherent evolution.

In Sec. II, we begin by recalling several common notions from classical community detection that we rely on in this work. This sets the stage for the development of a quantum treatment of community detection in Sec. III. We then turn to several examples in Sec. IV, including the LHCII complex mentioned previously, before concluding in Sec. V.

\section{COMMUNITY DETECTION}

Community detection is the partitioning of a set of nodes $\mathcal{N}$ into nonoverlapping [42] and nonempty subsets $\mathcal{A}, \mathcal{B}, \mathcal{C}, \ldots \subseteq \mathcal{N}$, called communities, that together cover $\mathcal{N}$.

There is usually no agreed upon optimal partitioning of nodes into communities. Instead, there is an array of approaches that differ in both the definition of optimality and the method used to achieve, exactly or approximately, this optimality (see Ref. [3] for a recent review). In classical networks, optimality is, for example, defined statistically [43], e.g., in terms of connectivity [1] or communicability $[44,45]$, or increasingly, and sometimes relatedly [46], in terms of stochastic random walks [47-49]. Our particular focus is on the latter, since the concept of transport (e.g., a quantum walk) is central to nearly all studies conducted in quantum physics. As for achieving optimality, methods include direct maximization via simulated annealing $[10,40]$ or, usually faster, iterative division or agglomeration of communities [50]. We focus on the latter since it provides a simple and effective way of revealing a full hierarchical structure of the network, requiring only the definition of the closeness of a pair of communities.

Formally, hierarchical community structure detection methods are based on a (symmetric) closeness function $c(\mathcal{A}, \mathcal{B})=c(\mathcal{B}, \mathcal{A})$ of two communities $\mathcal{A} \neq \mathcal{B}$. In the agglomerative approach, at the lowest level of the hierarchy, the nodes are each assigned their own communities. An iterative procedure then follows, in each step of which the closest pair of communities (maximum closeness c) are merged. This procedure ends at the highest level, where all nodes are in the same community. To avoid instabilities in this agglomerative procedure, the closeness function is required to be nonincreasing under the merging of two communities, $c(\mathcal{A} \cup \mathcal{B}, \mathcal{C}) \leq \max [c(\mathcal{A}, \mathcal{C}), c(\mathcal{B}, \mathcal{C})]$, which allows the representation of the community structure as a linear hierarchy indexed by the merging closeness. The resulting structure is often represented as a dendrogram (as shown in Fig. 1) [51].

This leaves open the question of which level of the hierarchy yields the optimal community partitioning. If a partitioning is desired for simulation, for example, then there may be a desired maximum size or minimum number of communities. However, without such constraints, one can still ask what is the best choice of communities within those given by the hierarchical structure.

A type of measure that is often used to quantify the quality of a community partitioning choice for this purpose is modularity [53-55], denoted $Q$. It was originally introduced in the classical network setting, in which a network is specified by a (symmetric) adjacency matrix of (non-negative) elements $A_{i j}=A_{j i} \geq 0\left(A_{i i}=0\right)$, each off-diagonal element giving the weight of connections between nodes $i$ and $j \neq i$ [56]. The modularity attempts to measure the fraction of weights connecting elements in the same community, relative to what might be expected. Specifically, one takes the fraction of intracommunity weights and subtracts the average fraction obtained when the start and end points of the connections are reassigned randomly, subject to the constraint that the total

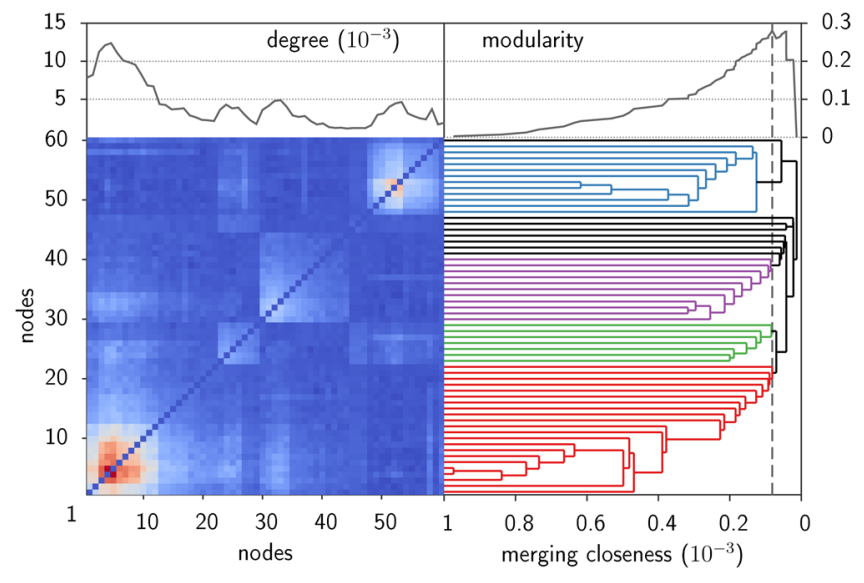

FIG. 1. Hierarchical community structure arising from a quantum evolution. On the left are the closenesses $c(i, j)$ between $n=60$ nodes. On the right is the dendrogram showing the resulting hierarchical community structure. The dashed line shows the optimum level within this hierarchy, according to the modularity. The particular example shown here is the one corresponding to Fig. 3(d). 
connectivity $k_{i}=\sum_{j} A_{i j}$ of each node is fixed. The modularity is then given by

$$
Q=\frac{1}{2 m} \operatorname{tr}\left\{C^{T} B C\right\}
$$

where $m=\frac{1}{2} \sum_{i} k_{i}$ is the total weight of connections, $B$ is the modularity matrix with elements $B_{i j}=A_{i j}-k_{i} k_{j} / 2 m$, and $C$ is the community matrix, with elements $C_{i \mathcal{A}}$ equal to unity if $i \in \mathcal{A}$, otherwise zero. The modularity then takes values strictly less than one, possibly negative, and exactly zero in the case that the nodes form a single community.

As we see, there is no natural adjacency matrix associated with the quantum network, and so, for the purposes of modularity, we use $A_{i j}=c(i, j)$ for $i \neq j$. The modularity $Q$ thus measures the fraction of the closeness that is intracommunity, relative to what would occur if the internode closeness $c(i, j)$ were randomly mixed while fixing the total closeness $k_{i}=\sum_{j \neq i} c(i, j)$ of each node to all others. Thus, both the community structure and optimum partitioning depend solely on the choice of the closeness function.

Modularity-based methods such as those noted above are intuitive, fast, and for the most part effective, yet we must note that for classical systems it has been shown that modularity-based methods suffer from a number of flaws that influence the overall efficacy of those approaches. In Refs. [40,41], modularity-based methods show a poor performance in large, sparse real-world and model networks. This is due mainly to the resolution limit problem [57], where small communities can be overlooked, and modularity landscape degeneracy, which strongly influence accuracy in large networks. Another modularity-related problem is the so-called detectability-undetectability threshold [58-60] where an approximate bipartition of the system becomes undetectable in some cases, in particular, in the presence of degree homogeneity. However, in the present work we focus on quantum networks whose size typically remains small compared to classical targets of community detection algorithms, and for which the derived adjacency matrices are not sparse. These characteristics help to limit the known flaws of our modularity-based approach, making it adequate for our purposes.

Finally, once a community partitioning is obtained, it is often desired to compare it against another. Here, we use the common normalized mutual information (NMI) [61-63] as a measure of the mutual dependence of two community partitionings. Each partitioning $X=\{\mathcal{A}, \mathcal{B}, \ldots\}$ is represented by a probability distribution $P_{X}=$ $\{|\mathcal{A}| /|\mathcal{N}|\}_{\mathcal{A} \in X}$, where $|\mathcal{A}|=\sum_{i} C_{i \mathcal{A}}$ is the number of nodes in community $\mathcal{A}$. The similarity of two community partitionings $X$ and $X^{\prime}$ depends on the joint distribution $P_{X X^{\prime}}=\left\{\left|\mathcal{A} \cap \mathcal{A}^{\prime}\right| /|\mathcal{N}|\right\}_{\mathcal{A} \in X, \mathcal{A}^{\prime} \in X^{\prime}}, \quad$ where $\quad\left|\mathcal{A} \cap \mathcal{A}^{\prime}\right|=$ $\sum_{i} C_{i \mathcal{A}} C_{i \mathcal{A}^{\prime}}$ is the number of nodes that belong to both communities $\mathcal{A}$ and $\mathcal{A}^{\prime}$. Specifically, NMI is defined as

$$
\operatorname{NMI}\left(X, X^{\prime}\right)=\frac{2 I\left(X, X^{\prime}\right)}{H(X)+H\left(X^{\prime}\right)} .
$$

Here, $H(X)$ is the Shannon entropy of $P_{X}$, and the mutual information $I\left(X, X^{\prime}\right)=H(X)+H\left(X^{\prime}\right)-H\left(X, X^{\prime}\right)$ depends on the entropy $H\left(X, X^{\prime}\right)$ of the joint distribution $P_{X X^{\prime}}$. The mutual information is the average of the amount of information about the community of a node in $X$ obtained by learning its community in $X^{\prime}$. The normalization ensures that the NMI has a minimum value of zero and takes its maximum value of unity for two identical community partitionings. The symmetry of the definition of NMI follows from that of mutual information and Eq. (2).

\section{QUANTUM COMMUNITY DETECTION}

The task of community detection has a particular interpretation in a quantum setting. The state of a quantum system is described in terms of a Hilbert space $\mathcal{H}$, spanned by a complete orthonormal set of basis states $\{|i\rangle\}_{i \in \mathcal{N}}$. Each basis state $|i\rangle$ can be associated with a node $i$ in a network and often, as in the case of single exciton transport, there is a clear choice of basis states that makes this abstraction to a spatially distributed network natural.

The partitioning of nodes into communities then corresponds to the partitioning of the Hilbert space $\mathcal{H}=\bigoplus_{\mathcal{A} \in X} \mathcal{V}_{\mathcal{A}}$ into mutually orthogonal subspaces $\mathcal{V}_{\mathcal{A}}=\operatorname{span}_{i \in \mathcal{A}}\{|i\rangle\}$. As with classical networks, one can then imagine an assortment of optimality objectives for community detection, for example, to identify a partitioning into subspaces in which intersubspace transport is small, or in which the state of the system remains relatively unchanged within each subspace. In the next two subsections, we introduce two classes of community closeness measures that correspond to these objectives. Technical details can be found in the Supplemental Material [64].

In what follows, we focus our analysis on an isolated quantum system governed by Hamiltonian $H$, which enables us to derive convenient closed-form expressions for the closeness measures. We may expand $H$ in the node basis $\{|i\rangle\}_{i \in \mathcal{N}}$ :

$$
H=\sum_{i j} H_{i j}|i\rangle\langle j|
$$

A diagonal element $H_{i i}$ is a real value denoting the energy of state $|i\rangle$, while an off-diagonal element $H_{i j}, i \neq j$ is a complex weight denoting the change in the amplitude of the wave function during a transition from state $|j\rangle$ to $|i\rangle$. The matrix formed by these elements can be thought of as a $|\mathcal{N}| \times|\mathcal{N}|$ complex adjacency matrix. Within the requirement of being Hermitian, in quantum mechanics the fact that the Hamiltonian may have complex elements leads to a range of phenomena not captured by real matrices, such as time-reversal symmetry breaking $[19,65]$. In the case that 
each $|i\rangle$ corresponds to a particle being localized at some node $i$ that is separated from the others, the Hamiltonian describes a single-particle spinless walk with an energy landscape given by the diagonal elements and transition amplitudes by the off-diagonal elements. Any quantum evolution can be viewed in this picture, making the singleparticle spinless walk scenario rather general.

A community partitioning based on a Hamiltonian $H$ could be used, among other things, to guide the simulation or analysis of a more complete model in the presence of an environment, where this more complete model may be much more difficult to describe. Additionally, our method could be generalized to use closeness measures based on open-system dynamics obtained numerically.

\section{A. Intercommunity transport}

Several approaches to detecting communities in classical networks are based on the flow of probability through the network during a classical random walk [46-49,66,67]. In particular, many of these methods seek communities for which the intercommunity probability flow or transport is small. A natural approach to quantum community detection is thus to consider the flow of probability during a continuous-time quantum walk and to investigate the change in the probability of observing the walker within each community:

$T_{X}(t)=\sum_{\mathcal{A} \in X} T_{\mathcal{A}}(t)=\sum_{\mathcal{A} \in X} \frac{1}{2}\left|p_{\mathcal{A}}\{\rho(t)\}-p_{\mathcal{A}}\{\rho(0)\}\right|$,

where $\rho(t)=e^{-\mathrm{i} H t} \rho(0) e^{\mathrm{i} H t}$ is the state of the walker, at time $t$, during the walk generated by $H$, and

$$
p_{\mathcal{A}}\{\rho\}=\operatorname{tr}\left\{\Pi_{\mathcal{A}} \rho\right\}
$$

is the probability of a walker in state $\rho$ being found in community $\mathcal{A}$ upon a von Neumann-type measurement [68] $\Pi_{\mathcal{A}}=\sum_{i \in \mathcal{A}}|i\rangle\langle i|$ is the projector to $\mathcal{A}$ subspace.

The initial state $\rho(0)$ can be chosen freely. The change in intercommunity transport is clearest when the process begins either entirely inside or entirely outside each community. Because of this, we choose the walker to be initially localized at a single node $\rho(0)=|i\rangle\langle i|$ and then, for symmetry, sum $T_{X}(t)$ over all $i \in \mathcal{N}$. This results in the particularly simple expression

$$
T_{\mathcal{A}}(t)=\sum_{i \in \mathcal{A}, j \notin \mathcal{A}} \frac{R_{i j}(t)+R_{j i}(t)}{2}=\sum_{i \in \mathcal{A}, j \notin \mathcal{A}} \tilde{R}_{i j}(t),
$$

where $R(t)$ is the doubly stochastic transfer matrix whose elements $R_{i j}(t)=\left|\left\langle i\left|e^{-\mathrm{i} H t}\right| j\right\rangle\right|^{2}$ give the probability of transport from node $j$ to node $i$, and $\tilde{R}(t)$ its symmetrization. This is reminiscent of classical community detection methods, e.g., Ref. [49], using closeness measures based on the transfer matrix of a classical random walk.

We can thus build a community structure that seeks to reduce $T_{X}(t)$ at each hierarchical level by using the closeness function

$$
\begin{aligned}
c_{t}^{T}(\mathcal{A}, \mathcal{B}) & =\frac{T_{\mathcal{A}}(t)+T_{\mathcal{B}}(t)-T_{\mathcal{A} \cup \mathcal{B}}(t)}{|\mathcal{A}||\mathcal{B}|} \\
& =\frac{2}{|\mathcal{A}||\mathcal{B}|} \sum_{i \in \mathcal{A}, j \in \mathcal{B}} \tilde{R}_{i j}(t),
\end{aligned}
$$

where the numerator is the decrement in $T_{X}(t)$ caused by merging communities $\mathcal{A}$ and $\mathcal{B}$. The normalizing factor in Eq. (7) avoids the effects due to the uninteresting scaling of the numerator with the community size.

Since a quantum walk does not converge to a stationary state, a time average of the closeness defined in Eq. (7) is needed to obtain a quantity that eventually converges with increasing time. Given the linearity of the formulation, this corresponds to replacing the transport probability $R_{i j}(t)$ in Eq. (7) with its time average:

$$
\hat{R}_{i j}(t)=\frac{1}{t} \int_{0}^{t} R_{i j}\left(t^{\prime}\right) d t^{\prime}
$$

It follows that, as with similar classical community detection methods [47], our method is, in fact, a class of approaches, each corresponding to a different time $t$. The appropriate value of $t$ depends on the specific application; for example, a natural time scale might be the decoherence time. Not wishing to lose generality and focus on a particular system, we focus here on the short- and longtime limits.

In the short-time limit $t \rightarrow 0$, relevant if $t H_{i j} \ll 1$ for $i \neq j$, the averaged transfer matrix $\hat{T}_{i j}(t)$ is simply proportional to $\left|H_{i j}\right|^{2}$. Note that in the short-time limit there is no interference between different paths from $|i\rangle$ to $|j\rangle$, and therefore, for short times $c_{t}^{T}(i, j)$ does not depend on the on-site energies $H_{i i}$ or the phases of the hopping elements $H_{i j}$. This is because, to leading order in time, interference does not play a role in the transport out of a single node. For this reason, we refer to this approach as "semiclassical."

In the long-time limit $t \rightarrow \infty$, relevant if $t$ is much larger than the inverse of the smallest gap between distinct eigenvalues of $H$, the probabilities are elements of the mixing matrix [69],

$$
\lim _{t \rightarrow \infty} \hat{R}_{i j}(t)=\sum_{k}\left|\left\langle i\left|\Lambda_{k}\right| j\right\rangle\right|^{2},
$$

where $\Lambda_{k}$ is the projector onto the $k$ th eigenspace of $H$. This thus provides a simple spectral method for building the community structure.

Note that, unlike in a classical infinitesimal stochastic walk where each $\hat{R}_{i j}(t)$ eventually becomes proportional to 
the connectivity $k_{j}$ of the final node $j$, the long-time limit in the quantum setting is nontrivial and, as we see, $\hat{R}_{i j}(t)$ retains a strong impression of the community structure for large $t$ [70].

\section{B. Intracommunity fidelity}

Classical walks, and the community detection methods based on them, are fully described by the evolution of the probabilities of the walker occupying each node. The previous quantum community detection approach is based on the evolution of the same probabilities but for a quantum walker. However, quantum walks are richer than this; they are not fully described by the evolution of the node-occupation probabilities. We therefore introduce another community detection method that captures the full quantum dynamics within each community subspace.

Instead of reducing merely the change in probability within the community subspaces, we reduce the change in the projection of the quantum state in the community subspaces. This change is measured using (squared) fidelity, a common measure of distance between two quantum states. For a walk beginning in state $\rho(0)$, we therefore focus on the quantity

$$
F_{X}(t)=\sum_{\mathcal{A} \in X} F_{\mathcal{A}}(t)=\sum_{\mathcal{A} \in X} F^{2}\left\{\Pi_{\mathcal{A}} \rho(t) \Pi_{\mathcal{A}}, \Pi_{\mathcal{A}} \rho(0) \Pi_{\mathcal{A}}\right\},
$$

where $\Pi_{\mathcal{A}} \rho \Pi_{\mathcal{A}}$ is the projection of the state $\rho$ onto the subspace $\mathcal{V}_{\mathcal{A}}$ and

$$
F\{\rho, \sigma\}=\operatorname{tr}\{\sqrt{\sqrt{\rho} \sigma \sqrt{\rho}}\} \in[0, \sqrt{\operatorname{tr}\{\rho\} \operatorname{tr}\{\sigma\}}]
$$

is the fidelity, which is symmetric between $\rho$ and $\sigma$.

We build a community structure that seeks to maximize the increase in $F_{X}(t)$ at each hierarchical level by using the closeness measure

$$
c_{t}^{F}(\mathcal{A}, \mathcal{B})=\frac{F_{\mathcal{A} \cup \mathcal{B}}(t)-F_{\mathcal{A}}(t)-F_{\mathcal{B}}(t)}{|\mathcal{A}||\mathcal{B}|} \in[-1,1],
$$

i.e., the change in $F_{X}(t)$ caused by merging communities $\mathcal{A}$ and $\mathcal{B}$. Our choice for the denominator prevents uninteresting size scaling, as in Eq. (7).

The initial state $\rho(0)$ can be chosen freely. Here, we choose the pure uniform superposition state $\rho(0)=$ $\left|\psi_{0}\right\rangle\left\langle\psi_{0}\right|$ satisfying $\left\langle i \mid \psi_{0}\right\rangle=1 / \sqrt{n}$ for all $i$. This state was used to investigate the effects of the connectivity on the dynamics of a quantum walker in Ref. [18].

As for our other community detection approach, we consider the time average of Eq. (12), which yields

$$
c_{t}^{F}(\mathcal{A}, \mathcal{B})=\frac{2}{|\mathcal{A}||\mathcal{B}|} \sum_{i \in \mathcal{A}, j \in \mathcal{B}} \operatorname{Re}\left[\hat{\rho}_{i j}(t) \rho_{j i}(0)\right],
$$

where $\hat{\rho}_{i j}(t)=(1 / t) \int_{0}^{t} d t^{\prime} \rho_{i j}\left(t^{\prime}\right)$. In the long-time limit, the time average of the density matrix takes a particularly simple expression:

$$
\lim _{t \rightarrow \infty} \hat{\rho}_{i j}(t)=\sum_{k} \Lambda_{k} \rho_{i j}(0) \Lambda_{k}
$$

where $\Lambda_{k}$ is the same as in Sec. III A.

The definition of community closeness given in Eq. (12) can exhibit negative values. In this case, the usual definition of modularity fails [71] and one must extend it. In this work, we use the definition of modularity proposed in Ref. [71], which coincides with Eq. (1) in the case of nonnegative closeness. The extended definition treats negative and positive links separately, and tries to minimize intracommunity negative links while maximizing intracommunity positive links.

\section{PERFORMANCE ANALYSIS}

To analyze the performance of our quantum community detection methods, we apply them to three different networks. The first one (Sec. IVA) is a simple quantum network, which we use to highlight how some intuitive notions in classical community detection do not necessarily transfer over to quantum systems. The second example (Sec. IV B) is an artificial quantum network designed to exhibit a clear classical community structure, which we show is different from the quantum community structure obtained and fails to capture significant changes in this structure induced by quantum mechanical phases on the hopping elements of the Hamiltonian. The final network (Sec. IV C) is a real-world quantum biological network, describing the LHCII light-harvesting complex, for which we find a consistent quantum community structure differing from the community structure cited in the literature. These findings confirm that a quantum mechanical treatment of community detection is necessary as classical and semiclassical methods cannot reproduce the structures that appropriately capture quantum effects.

Below, we compare quantum community structures against more classical community structures, such the one given by the semiclassical method based on the short-time transport and, in the case of the example of Sec. IV B, the classical network from which the quantum network is constructed. Additionally we use a traditional classical community detection algorithm OSLOM [43], an algorithm based on the maximization of the statistical significance of the proposed partitioning, whose input adjacency matrix $A$ must be real. For this purpose, we use the absolute values of the Hamiltonian elements in the site basis: $A_{i j}=\left|H_{i j}\right|$.

\section{A. Simple quantum network}

Here, we use a simple six-site network model to study ways in which quantum effects lead to nonintuitive results 
and how methods based on different quantum properties can, accordingly, lead to very different choices of communities.

We begin with two disconnected cliques of three nodes each, where all Hamiltonian matrix elements within the groups are identical and real. Figure 2 illustrates this highly symmetric topology. The community detection method based on quantum transport identifies the two fully connected groups as two separate communities [Fig. 2(a)], as is expected. In contrast, the methods based on fidelity predict counterintuitively only a single community; two disconnected nodes can retain coherence and, by this measure, be considered part of the same community [Fig. 2(b)].

This symmetry captured by the fidelity-based community structure breaks down if we introduce random perturbations into the Hamiltonian. Specifically, the fidelity-based

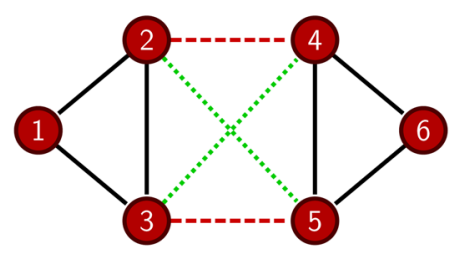

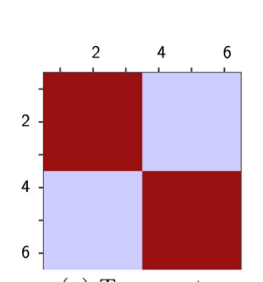

(a) Transport

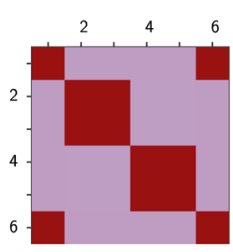

(d) Coherent phases

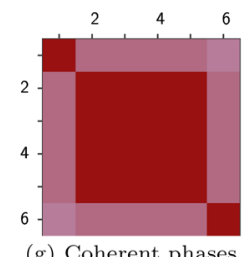

(g) Coherent phases
Disconnected components:

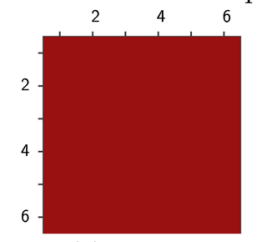

(b) Fidelity

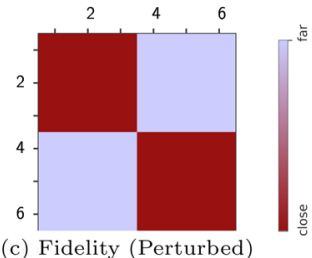

(c) Fidelity (Perturbed)
Phases' effect on transport:

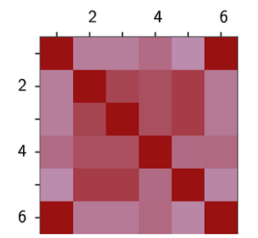

(e) Random phases

Phases' effect on fidelity:

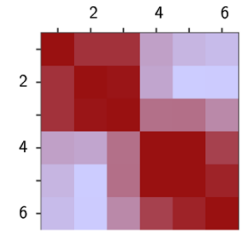

(h) Random phases

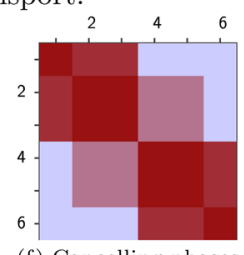

(f) Cancelling phases

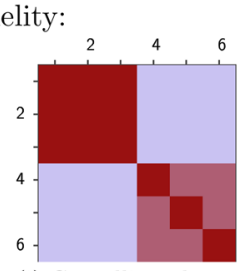

(i) Cancelling phases
FIG. 2. Simple quantum network - a graph with six nodes. Each solid line represents transition amplitude $H_{i j}=1$. For dashed and dotted lines, the transition amplitude can be either zero (a)-(c) or the absolute value is the same $\left|H_{i j}\right|=1$ but phase is $(\mathrm{d}),(\mathrm{g})$ coherent (all ones), (e),(h) random $\exp \left(i \varphi_{k}\right)$ for each link, or (f),(i) canceling (1 for dashed red and -1 for dotted green). Plots show the node closeness for both methods based on transport and fidelity (only the long-time averages are considered). [In plots (g)-(i) we use a perturbed Hamiltonian to solve the degeneracy of the eigenvalues; this explains the nonsymmetric closeness in (i)]. closeness $c_{t}^{F}$ is sensitive to perturbations of the order $t^{-1}$, above which the community structure is divided into the two groups of three [Fig. 2(c)] expected from transport considerations. Thus, we may tune the resolution of this community structure method to asymmetric perturbations by varying $t$.

Because of quantum interference, we expect that the Hamiltonian phases should significantly affect the quantum community partitioning. The same toy model can be used to demonstrate this effect. For example, consider adding four elements to the Hamiltonian corresponding to hopping from nodes 2 and 3 to 4 and 5 (see diagram in Fig. 2). If these hopping elements are all identical to the others, it is the two nodes, 1 and 6 , that are not directly connected for which the internode transport is largest (and thus their internode closeness is the largest). However, when the phases of the four additional elements are randomized, this transport is decreased. Moreover, when the phases are canceling, the transport between nodes 1 and 6 is reduced to zero, and the closeness between them is minimized [see Figs. 2(d)-2(f).

The fidelity method has an equally strong dependence on the phases [see Figs. 2(g)-2(i), with variations in the phases breaking up the network from a large central community (with nodes 1 and 6 alone) into the two previously identified communities.

\section{B. Artificial quantum network}

The Hamiltonian of our second quantum network is constructed from the adjacency matrix $A$ of a classical unweighted, undirected network exhibiting a clear classical partitioning, using the relation $H_{i j}=A_{i j}$. We construct $A$ using the algorithm proposed by Lancichinetti et al. in Ref. [72], which provides a method to construct a network with heterogeneous distribution both for the node degree and for the communities' dimension and a controllable inter-community connection. We start with a rather small network of 60 nodes with average intracommunity connectivity $\langle k\rangle=6$, and only $5 \%$ of the edges are rewired to join communities. The network is depicted in Fig. 3(a). To confirm the expected, the known classical community structure is indeed obtained by the semiclassical short-timetransport algorithm [73] and the OSLOM algorithm [see Figs. 3(b) $-3(\mathrm{e})$, achieving NMI $=0.953$ and NMI $=0.975$ with the known structure, respectively.

The quantum methods based on the long-time average of both transport and fidelity reproduce the main features of the original community structure while unveiling new characteristics. The transport-based long-time average method (NMI $=0.82$ relative to the classical partitioning) exhibits disconnected communities; i.e., the corresponding subgraph is disconnected. This behavior can be explained by interference-enhanced quantum walker dynamics, as exhibited by the toy model in the previous subsection. The long-time average fidelity method $(\mathrm{NMI}=0.85)$ returns the four main classical communities plus a number of 


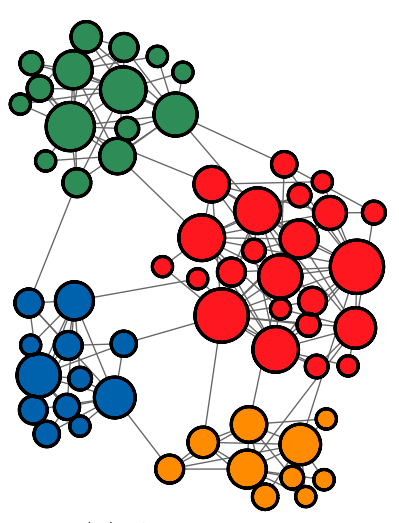

(a) Original data

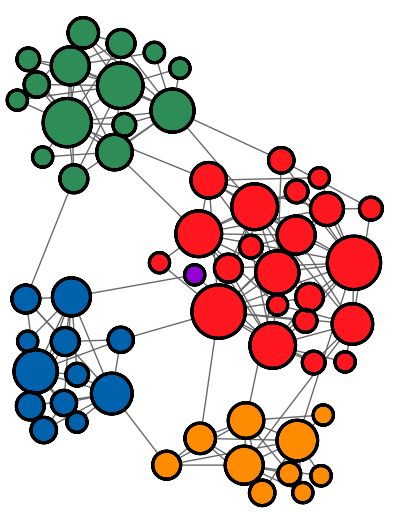

(e) OSLOM

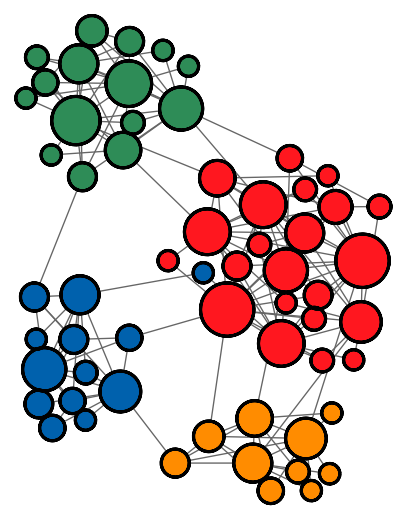

(b) Transport; $t \rightarrow 0$

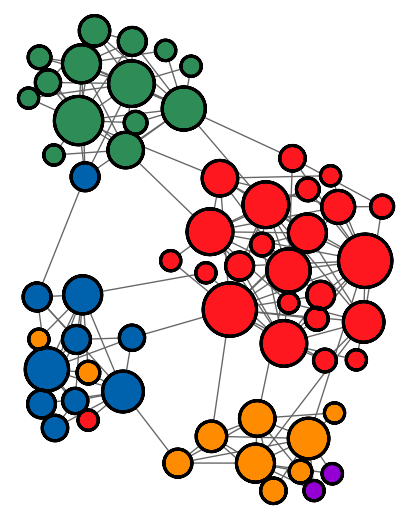

(c) Transport; $t \rightarrow \infty$

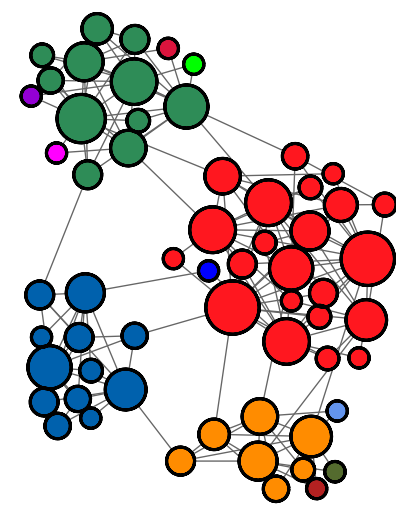

(d) Fidelity; $t \rightarrow \infty$

Dependence on phases:

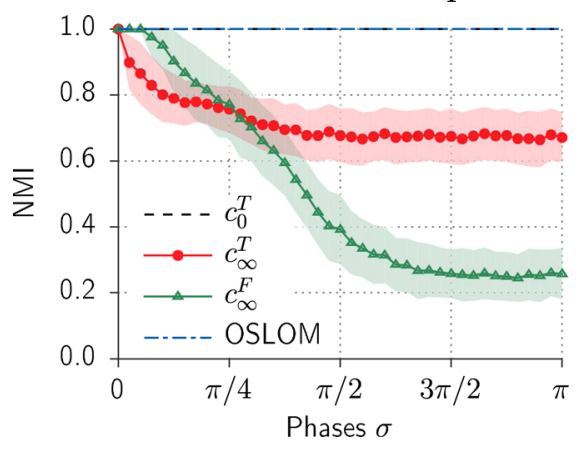

(f) Original partitioning

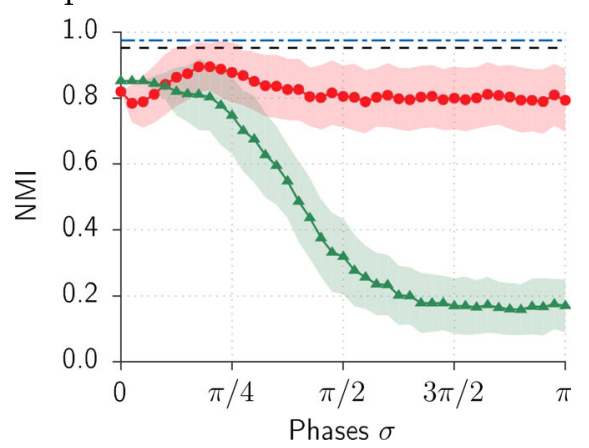

(g) Classical model

FIG. 3. Artificial community structure. (a) Classical community structure used in creating the network. (b)-(e) Community partitionings found using the three quantum methods and OSLOM. (f),(g) Behavior of the approaches as the phases of the Hamiltonian elements are randomly sampled from a Gaussian distribution of width $\sigma$. The mean NMI, compared with zero phase partitioning (f) and the classical model data (g), over 200 samplings of the phase distribution is plotted. The standard deviation is indicated by the shading. Both OSLOM and $c_{0}^{T}$ are insensitive to phases and thus do not respond to the changes in the Hamiltonian.

single-node communities. Both methods demonstrate that the quantum and classical community structures are unsurprisingly different, with the quantum community structure clearly dependent on the quantum property being optimized, more so than the different classical partitionings.

\section{Adjusted phases}

As shown in Sec. IV A, due to interference, the dynamics of the quantum system can change drastically if the phases of the Hamiltonian elements are nonzero. This is known as a chiral quantum walk [19]. Such walks exhibit, for example, time-reversal symmetry breaking of transport between sites [19], and it has been proposed that nature might actually make use of phase-controlled interference in transport processes [74]. OSLOM, our semiclassical shorttime transport algorithm, and other classical community partitioning methods are insensitive to changes in the hopping phases. Thus, by establishing that the quantum community structure is sensitive to such changes in phase, as expected from above, we show that classical methods are inadequate for finding quantum community structure.
To analyze this effect, we take the previous network and adjust the phases of the Hamiltonian terms while preserving their absolute values. Specifically, the phases are sampled randomly from a normal distribution with mean zero and standard deviation $\sigma$. We find that, typically, as the standard deviation $\sigma$ increases, when comparing quantum communities and the corresponding communities without phases, the NMI between them decreases, as shown in Fig. 3(f). A similar deviation reflects on the comparison with the classical communities used to construct the system, shown in Fig. 3(g). This sensitivity of the quantum community structures to phases, as revealed by the NMI, confirms the expected inadequacy of classical methods. The partitioning based on long-time average fidelity seems to be the most sensitive to phases.

\section{Light-harvesting complex}

An increasing number of biological networks of nontrivial topology are being described using quantum mechanics. For example, light-harvesting complexes have 

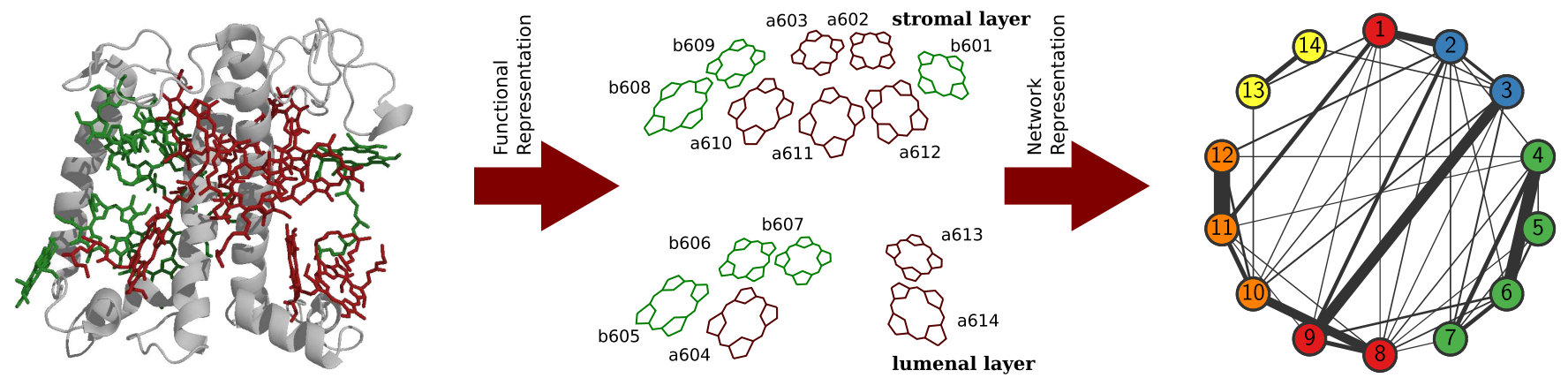

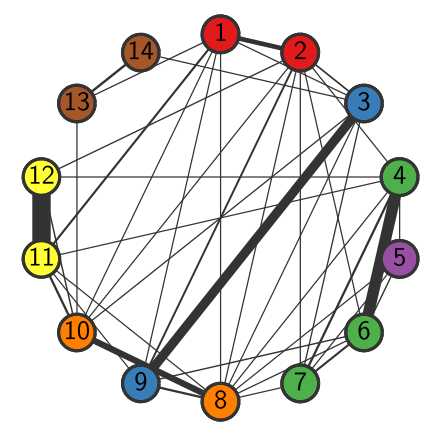

(a) Transport; $t \rightarrow 0$

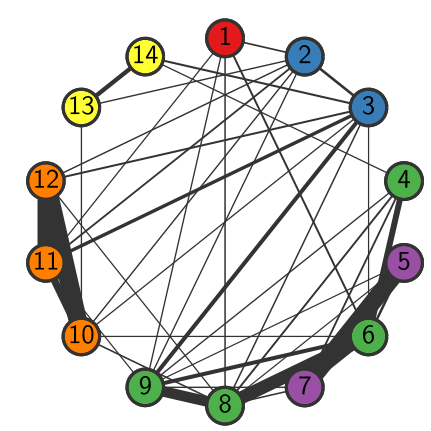

(b) Transport; $t \rightarrow \infty$

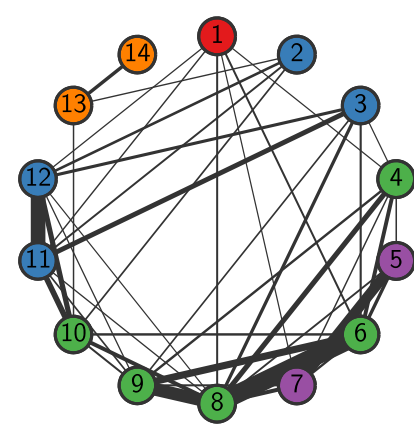

(c) Fidelity; $t \rightarrow \infty$

FIG. 4. Light-harvesting complex II (LHCII). (Top, left) Monomeric subunit of the LHCII complex with pigments Chl-a (red) and Chl-b (green) packed in the protein matrix (gray). (Top, center) Schematic representation of Chl-a and Chl-b in the monomeric subunit; here, the labeling follows the usual nomenclature (b601, a602,...). (Top, right) Network representation of the pigments in circular layout; colors represent the typical partitioning of the pigments into communities. The widths of the links represent the strength of the couplings $\left|H_{i j}\right|$ between nodes. Here, the labels maintain only the ordering $(b 601 \rightarrow 1, a 602 \rightarrow 2, \ldots)$. (a)-(c) Quantum communities as found by the different quantum community detection methods. Link width denotes the pairwise closeness of the nodes.

drawn significant attention in the quantum information community.

One of these is the LHCII, a two-layer 14-chromophore complex embedded into a protein matrix (see Fig. 4 for a sketch) that collects light energy and directs it toward the reaction center where it is transformed into chemical energy. The system can be described as a network of 14 sites connected with a nontrivial topology. The singleexciton subspace is spanned by 14 basis states, each corresponding to a node in the network, and the Hamiltonian in this basis was found in Ref. [38].

In a widely adopted chromophore community structure [37], the sites are partitioned by hand into communities according to their physical closeness (e.g., there are no communities spanning the two layers of the complex) and the strength of Hamiltonian couplings (see the top right of Fig. 4). Here, we apply our ab initio automated quantum community detection algorithms to the same Hamiltonian.

All of our approaches predict a modified partitioning to that commonly used in the literature. The method based on short-time transport returns communities that do not connect the two layers. This semiclassical approach relies only on the coupling strength of the system, without considering interference effects, and provides the closest partitioning to the one provided by the literature (also relying only on the coupling strengths). Meanwhile, the methods based on the long-time transport and fidelity return very similar community partitionings, in which node 6 on one layer and node 9 on the other are in the same community. These two long-time community partitionings are identical, except one of the communities predicted by the fidelity-based method is split when using the transport-based method. It is, therefore, a difference in modularity only.

The classical OSLOM algorithm fails spectacularly: it gives only one significant community involving nodes 11 and 12 , which exhibit the highest coupling strength. If assigning a community to each node is forced, a unique community with all nodes is provided.

Note that here we have used the LHCII closed-system dynamics, valid only for short times, to partition it. As explained in Sec. III, for the purpose of analysis, one could alternatively use the less tractable open-system dynamics to obtain a partitioning that reflects the environment of the LHCII [26]. However, we argue that community partitioning, e.g., that based on the closed-system dynamics, is essential in devising approaches to simulating the full opensystem dynamics.

\section{DISCUSSION}

We develop methods to detect community structure in quantum systems, thereby extending the purview of 
community detection from classical networks to include quantum networks. Our approach involves the development of a number of methods that focus on different characteristics of the system and return a community structure reflecting that specific characteristic. The variation of the quantum community structure with the property on which this structure is based seems greater than for classical community structures.

All of our methods are based on the full unitary dynamics of the system, as described by the Hamiltonian, and account for quantum effects such as coherent evolution and interference. In fact, phases are often fundamental to characterizing the system evolution. For example, Harel and Engel [74] have shown that in light-harvesting complexes interference between pathways is important even at room temperature. In our light-harvesting complex example (see Sec. IV C), the $a b$ initio community structures provided by the long-time measures propose consistent communities that stretch across the lumenal and stromal layers of the complex, absent in the structure proposed by the community.

Since we consider time evolution, the averaging time $t$ acts as a tuning parameter for the partitioning methods. In the case of transport, it transforms the method from a semiclassical approach $(t \rightarrow 0)$ to a fully quantum-aware measure $(t \rightarrow \infty)$, For all times, the complexity of our algorithms scales polynomially in the number of nodes $|\mathcal{N}|$, at worst $O\left(|\mathcal{N}|^{3}\right)$ if the diagonalization of $H$ is required. This allows the study of networks with node numbers up the thousands and tens of thousands, which is appropriate for the real-world quantum networks currently being considered.

As with classical community structure, there are many possible definitions of a quantum community. We restrict ourselves to two broad classes based on transport and fidelity under coherent evolution, both based on dynamics, though in the limits considered in this paper, the closenesses and thus quantum community structure can be expressed purely in terms of static properties. We end by briefly discussing some other possible definitions based on statics (the earliest classical community definitions were based on statics [75]). The first type is based on some quantum state $|\psi\rangle$, e.g., the ground state of $H$. We might wish to partition the network by repeatedly diving the network in two based on minimally entangled bipartitions. This could be viewed as identifying optimum communities for some cluster-based mean-field-like simulation [31] whose entanglement structure is expected to be similar to $|\psi\rangle$. The second type is based directly on the spectrum of the Hamiltonian $H$. We might partition the Hilbert space into unions of the eigenspaces of $H$ by treating the corresponding eigenvalues as 1D coordinates and applying a traditional agglomerative or divisive clustering algorithm on them. Note that the resulting partitioning would normally not be in the position basis.
The use of community detection in quantum systems addresses an open challenge in the drive to unite quantum physics and complex network science, and we expect such partitioning, based on our definitions or extensions such as above, to be used extensively in making the large quantum systems currently being targeted by quantum physicists tractable to numerical analysis. Conversely, quantum measures have also been shown to add novel perspectives to classical network analysis [76].

\section{ACKNOWLEDGMENTS}

We thank Michele Allegra, Leonardo Banchi, Giovanni Petri, and Zoltán Zimborás for fruitful discussions. M. F., T. H. J., and J.D.B. completed part of this study while visiting the Institute for Quantum Computing, at the University of Waterloo. P. M. acknowledges the Spanish MINCIN/MINECO project TOQATA (FIS2008-00784), EU Integrated Projects AQUTE and SIQS, and HISTERA project DIQUIP. T. H. J. acknowledges the European Research Council under the European Union's Seventh Framework Programme (FP7/2007-2013)/ERC Grant Agreement No. 319286 and the National Research Foundation and the Ministry of Education of Singapore for support. J. D. B. acknowledges the Foundational Questions Institute (under Grant No. FQXi-RFP3-1322) for financial support. All authors acknowledge the Q-ARACNE project funded by the Fondazione Compagnia di San Paolo.

[1] M. Girvan and M. E. J. Newman, Community Structure in Social and Biological Networks, Proc. Natl. Acad. Sci. U.S.A. 99, 7821 (2002).

[2] M. A. Porter, J.-P. Onnela, and P. J. Mucha, Communities in Networks, Not. Am. Math. Soc. 56, 1082 (2009).

[3] S. Fortunato, Community Detection in Graphs, Phys. Rep. 486, 75 (2010).

[4] S. A. Rice, The Identification of Blocs in Small Political Bodies, Am. Pol. Sci. Rev. 21, 619 (1927).

[5] W. W. Zachary, An Information Flow Model for Conflict and Fission in Small Groups, Journal of anthropological research 33, 452 (1977).

[6] D. Lusseau and M. E. J. Newman, Identifying the Role That Animals Play in Their Social Networks, Proc. R. Soc. B 271, S477 (2004).

[7] P. F. Jonsson, T. Cavanna, D. Zicha, and P. A. Bates, Cluster Analysis of Networks Generated through Homology: Automatic Identification of Important Protein Communities Involved in Cancer Metastasis, BMC Bioinf. 7, 2 (2006).

[8] S. L. Pimm, The Structure of Food Webs, Theor. Popul. Biol. 16, 144 (1979).

[9] A. E. Krause, K. A. Frank, D. M. Mason, R. E. Ulanowicz, and W. W. Taylor, Compartments Revealed in Food-Web Structure, Nature (London) 426, 282 (2003).

[10] R. Guimera and L. A. Nunes Amaral, Functional Cartography of Complex Metabolic Networks, Nature (London) 433, 895 (2005). 
[11] P. Holme, M. Huss, and H. Jeong, Subnetwork Hierarchies of Biochemical Pathways, Bioinformatics 19, 532 (2003).

[12] G. William Flake, S. Lawrence, C. Lee Giles, and F. M. Coetzee, Self-Organization and Identification of Web Communities, Computer 35, 66 (2002).

[13] L. Gauvin, A. Panisson, and C. Cattuto, Detecting the Community Structure and Activity Patterns of Temporal Networks: A Non-negative Tensor Factorization Approach, PLoS One 9, e86028 (2014).

[14] M. Allegra and P. Giorda, Topology and Energy Transport in Networks of Interacting Photosynthetic Complexes, Phys. Rev. E 85, 051917 (2012).

[15] M. B. Plenio and S. F. Huelga, Dephasing-Assisted Transport: Quantum Networks and Biomolecules, New J. Phys. 10, 113019 (2008).

[16] J. Adolphs and T. Renger, How Proteins Trigger Excitation Energy Transfer in the FMO Complex of Green Sulfur Bacteria, Biophys. J. 91, 2778 (2006).

[17] J. C. Baez and J. Biamonte, A Course on Quantum Techniques for Stochastic Mechanics, arXiv:1209.3632.

[18] M. Faccin, T. Johnson, J. Biamonte, S. Kais, and P. Migdał, Degree Distribution in Quantum Walks on Complex Networks, Phys. Rev. X 3, 041007 (2013).

[19] Z. Zimboras, M. Faccin, Z. Kadar, J. Whitfield, B. Lanyon, and J. Biamonte, Quantum Transport Enhancement by Time-Reversal Symmetry Breaking, Sci. Rep. 3, 2361 (2013).

[20] T. H. Johnson, S. R. Clark, and D. Jaksch, What is a Quantum Simulator?, EPJ Quantum Technol. 1, 10 (2014).

[21] A. K. Ringsmuth, G. J. Milburn, and T. M. Stace, Multiscale Photosynthetic and Biomimetic Excitation Energy Transfer, Nat. Phys. 8, 562 (2012).

[22] M. Sarovar, A. Ishizaki, G. R. Fleming, and K. Birgitta Whaley, Quantum Entanglement in Photosynthetic LightHarvesting Complexes, Nat. Phys. 6, 462 (2010).

[23] A. Ishizaki and G. R. Fleming, Quantum Coherence in Photosynthetic Light Harvesting, Annu. Rev. Condens. Matter Phys. 3, 333 (2012).

[24] Y.-C. Cheng and G. R. Fleming, Dynamics of Light Harvesting in Photosynthesis, Annu. Rev. Phys. Chem. 60, 241 (2009).

[25] F. Caruso, A. W. Chin, A. Datta, S. F. Huelga, and M. B. Plenio, Highly Efficient Energy Excitation Transfer in Light-Harvesting Complexes: The Fundamental Role of Noise-Assisted Transport, J. Chem. Phys. 131, 105106 (2009).

[26] M. Mohseni, P. Rebentrost, S. Lloyd, and A. Aspuru-Guzik, Environment-Assisted Quantum Walks in Photosynthetic Energy Transfer, J. Chem. Phys. 129, 174106 (2008).

[27] L. Banchi, G. Costagliola, A. Ishizaki, and P. Giorda, An Analytical Continuation Approach for Evaluating Emission Lineshapes of Molecular Aggregates and the Adequacy of Multichromophoric Förster Theory, J. Chem. Phys. 138 184107 (2013).

[28] D. S. Rokhsar and B. G. Kotliar, Gutzwiller Projection for Bosons, Phys. Rev. B 44, 10328 (1991).

[29] W. Krauth, M. Caffarel, and J.-P. Bouchaud, Gutzwiller Wave Function for a Model of Strongly Interacting Bosons, Phys. Rev. B 45, 3137 (1992).

[30] K Sheshadri, H. R. Krishnamurthy, R. Pandit, and T. V. Ramakrishnan, Superfluid and Insulating Phases in an
Interacting-Boson Model: Mean-Field Theory and the RPA, Europhys. Lett. 22, 257 (1993).

[31] D.S. Lühmann, Cluster Gutzwiller Method for Bosonic Lattice Systems, Phys. Rev. A 87, 043619 (2013).

[32] F. Verstraete, V. Murg, and J. Ignacio Cirac, Matrix Product States, Projected Entangled Pair States, and Variational Renormalization Group Methods for Quantum Spin Systems, Adv. Phys. 57, 143 (2008).

[33] J. Ignacio Cirac and F. Verstraete, Renormalization and Tensor Product States in Spin Chains and Lattices, J. Phys. A 42, 504004 (2009).

[34] H. Wang, I. Pshenichnyuk, R. Härtle, and M. Thoss, Numerically Exact, Time-Dependent Treatment of Vibrationally Coupled Electron Transport in Single-Molecule Junctions, J. Chem. Phys. 135, 244506 (2011).

[35] L. Cao, S. Krönke, O. Vendrell, and P. Schmelcher, The Multi-Layer Multi-Configuration Time-Dependent Hartree Method for Bosons: Theory, Implementation, and Applications, J. Chem. Phys. 139, 134103 (2013).

[36] X. Pan, Z. Liu, M. Li, and W. Chang, Architecture and Function of Plant Light-Harvesting Complexes II, Curr. Opin. Struct. Biol. 23, 515 (2013).

[37] V. I. Novoderezhkin, M. A. Palacios, H. van Amerongen, and R. van Grondelle, Excitation Dynamics in the LHCII Complex of Higher Plants: Modeling Based on the $2.72 \AA$ Crystal Structure, J. Phys. Chem. B 109, 10493 (2005).

[38] G. S. Schlau-Cohen, T. R. Calhoun, N. S. Ginsberg, E. L. Read, M. Ballottari, R. Bassi, R. van Grondelle, and G. R. Fleming, Pathways of Energy Flow in LHCII from TwoDimensional Electronic Spectroscopy, J. Phys. Chem. B 113, 15352 (2009).

[39] G. Carlsson and F. Mémoli, Characterization, Stability and Convergence of Hierarchical Clustering Methods, J. Mach. Learn. Res. 99, 1425 (2010).

[40] R. Guimerà, M. Sales-Pardo, and L. A. Nunes Amaral, Modularity from Fluctuations in Random Graphs and Complex Networks, Phys. Rev. E 70, 025101 (2004).

[41] B. H. Good, Y.-A. de Montjoye, and A. Clauset, Performance of Modularity Maximization in Practical Contexts, Phys. Rev. E 81, 046106 (2010).

[42] We do not consider generalizations to overlapping communities here.

[43] A. Lancichinetti, F. Radicchi, J. J. Ramasco, and S. Fortunato, Finding Statistically Significant Communities in Networks, PLoS One 6, e18961 (2011).

[44] E. Estrada, Community detection based on network communicability, Chaos 21, 016103 (2011).

[45] E. Estrada and N. Hatano, Communicability Graph and Community Structures in Complex Networks, Appl. Math. Comput. 214, 500 (2009).

[46] M. Meila and J. Shi, in Proceedings of the Eighth International Workshop on Artificial Intelligence and Statistics (2001); http://www.gatsby.ucl.ac.uk/aistats/ aistats2001/index.html.

[47] J.-C. Delvenne, S. N. Yaliraki, and M. Barahona, Stability of Graph Communities across Time Scales, Proc. Natl. Acad. Sci. U.S.A. 107, 12755 (2010).

[48] M. Rosvall and C. T. Bergstrom, Multilevel Compression of Random Walks on Networks reveals Hierarchical Organi- 
zation in Large Integrated Systems, PLoS One 6, e18209 (2011).

[49] P. Pons and M. Latapy, Computing Communities in Large Networks Using Random Walks (Springer, New York, 2005), pp. 284-293.

[50] T. Hastie, R. Tibshirani, and J. Friedman, The Elements of Statistical Learning Theory (Springer, New York, 2001).

[51] In general, it may happen that more than one pair of communities is at the maximum closeness. In this case, the decision on which pair merges first can influence the structure of the dendrogram; see Refs. [39,52]. In Ref. [39], a permutation invariant formulation of the agglomerative algorithm is given, where more than two clusters can be merged at once. In our work, we use this formulation unless stated otherwise.

[52] A. K. Jain and R. C. Dubes, Algorithms for Clustering Data (Prentice-Hall, Englewood Cliffs, NJ, 1988).

[53] M. E. J. Newman and M. Girvan, Finding and Evaluating Community Structure in Networks, Phys. Rev. E 69, 026113 (2004).

[54] M. E. J. Newman, Fast Algorithm for Detecting Community Structure in Networks, Phys. Rev. E 69, 066133 (2004).

[55] A. Clauset, M. E. J. Newman, and C. Moore, Finding Community Structure in Very Large Networks, Phys. Rev. E 70, 066111 (2004).

[56] As will become apparent, we need only consider undirected networks without self-loops.

[57] S. Fortunato and M. Barthelemy, Resolution Limit in Community Detection, Proc. Natl. Acad. Sci. U.S.A. 104, 36 (2007).

[58] R. R. Nadakuditi and M. E. J. Newman, Graph Spectra and the Detectability of Community Structure in Networks, Phys. Rev. Lett. 108, 188701 (2012).

[59] F. Radicchi, Detectability of Communities in Heterogeneous Networks, Phys. Rev. E 88, 010801 (2013).

[60] F. Radicchi, A Paradox in Community Detection, Europhys. Lett. 106, 38001 (2014).

[61] L. N. F. Ana and A. K. Jain, in Proceedings of Robust data clustering (IEEE, New York, 2003), Vol. 2, pp. II-128-II133.

[62] A. Strehl and J. Ghosh, Cluster Ensembles-A Knowledge Reuse Framework for Combining Multiple Partitions, J. Mach. Learn. Res. 3, 583 (2003).
[63] L. Danon, A. Díaz-Guilera, J. Duch, and A. Arenas, Comparing Community Structure Identification, J. Stat. Mech. (2005) P09008.

[64] See Supplemental Material at http://link.aps.org/ supplemental/10.1103/PhysRevX.4.041012 for a detailed derivation of the closeness measures.

[65] D. Lu, J. D. Biamonte, J. Li, H. Li, T. H. Johnson, V. Bergholm, M. Faccin, Z. Zimborás, R. Laflamme, J. Baugh, and S. Lloyd, Chiral Quantum Walks, arXiv:1405.6209.

[66] K. Astrup Eriksen, I. Simonsen, S. Maslov, and K. Sneppen, Modularity and Extreme Edges of the Internet, Phys. Rev. Lett. 90, 148701 (2003).

[67] E. Weinan, T. Li, and E. Vanden-Eijnden, Optimal Partition and Effective Dynamics of Complex Networks, Proc. Natl. Acad. Sci. U.S.A. 105, 7907 (2008).

[68] Equivalently, $p_{\mathcal{A}}\{\rho\}$ is the norm of the projection (performed by projector $\Pi_{\mathcal{A}}$ ) of the state $\rho$ onto the community subspace $\mathcal{V}_{\mathcal{A}}$.

[69] C. Godsil, Average Mixing of Continuous Quantum Walks, arXiv:1103.2578.

[70] Note that, apart from small or large times $t$, there is no guarantee of symmetry $R_{i j}(t)=R_{j i}(t)$ in the transfer matrix for a given Hamiltonian. See Ref. [19]. Hamiltonians featuring this symmetry, e.g., those with real $H_{i j}$, are called time symmetric.

[71] V. A. Traag and J. Bruggeman, Community Detection in Networks with Positive and Negative Links, Phys. Rev. E 80, 036115 (2009).

[72] A. Lancichinetti, S. Fortunato, and F. Radicchi, Benchmark Graphs for Testing Community Detection Algorithms, Phys. Rev. E 78, 046110 (2008).

[73] In the case of short-time transport, a small perturbation was also added to the closeness function in order to break the symmetries of the system.

[74] E. Harel and G. S. Engel, Quantum Coherence Spectroscopy Reveals Complex Dynamics in Bacterial LightHarvesting Complex 2 (LH2), Proc. Natl. Acad. Sci. U.S.A. 109, 706 (2012).

[75] M. Newman, Networks: An Introduction (Oxford University Press, Oxford, UK, 2009).

[76] E. Sánchez-Burillo, J. Duch, J. Gómez-Gardeñes, and D. Zueco, Quantum Navigation and Ranking in Complex Networks, Sci. Rep. 2, 605 (2012). 